\title{
MujERES Y ORGANIZACIÓN SOCIAL EN LA GESTIÓN DEL AGUA PARA CONSUMO HUMANO Y USO DOMÉSTICO EN BERRIOZÁBAL, ChIAPAS
}

\author{
Women and social organization in the water management for human consumption \\ and domestic use in Berriozabal, Chiapas
}

\author{
Verónica Gutiérrez-Villalpando, Dominga Austreberta Nazar-Beutelspacher \\ Emma Zapata-Martelo, Julio Contreras-Utrera, Benito Salvatierra-Izaba
}

\begin{abstract}
Resumen: El estudio tiene como objetivo analizar la organización comunitaria en la gestión del agua para consumo humano y uso doméstico y el papel que juegan las mujeres en ella en las localidades urbanas y rurales ubicadas en las subcuencas Río Sabinal y Cañón del Sumidero en Berriozábal, Chiapas. Se encontró que el agua de los ríos, manantiales y vertientes es suficiente, pero en la época de sequía disminuye de manera considerable y no alcanza para cubrir los requerimientos de quienes se benefician de estas fuentes. Por ello, las mujeres tienen que asumir la responsabilidad de abastecer el agua que se utiliza en el hogar, lo que les genera más carga de trabajo. También observamos que las localidades con mayor desventaja en condiciones de vivienda, servicios y equipamiento son las que no cuentan con comité de agua, lo que sugiere menor organización, pese a que ésta podría ser fundamental para la gestión del agua.
\end{abstract}

Palabras clave: mujeres, organización social, gestión comunitaria, autogestión, comités de agua.

Abstract: The study has the main objective to analyze community organization in the area of water management for human consumption and domestic use and the role women play in this in urban and rural areas, in the watersheds of Río Sabinal and Cañón del Sumidero in Berriozabal, Chiapas. It was found that the water found in the rivers, springs and slopes is sufficient, but in the dry season this decreases considerably, and it doesn't meet the needs of those who benefit from these sources. Therefore, the women have to take responsibility to provide the water that is used in the home, which generates more workload. We also observed that the localities at most disadvantage in terms of housing, services and equipment conditions, are those that don't have a water management committee, which suggests less organization, despite the fact this could be fundamental for water management in communities.

Keywords: woman, social organization, community management, self-management, water committees.

\footnotetext{
Verónica Gutiérrez-Villalpando, doctora en Ecología y Desarrollo Sustentable por el Colegio de la Frontera Sur. Temas de especialización: género, agua, conservación de recursos naturales, servicios ecosistémicos, cambio climático y bosques. Correo electrónico: bioveros@hotmail.com, vgutierrez@ecosur.mx.

Dominga Austreberta Nazar-Beutelspacher, doctora en estudios del Desarrollo Rural por el Colegio de Postgraduados. Investigadora titular C en El Colegio de la Frontera Sur. Temas de especialización: género y salud, equidad y desarrollo, políticas de población. Correo electrónico: anazar@ecosur.mx.

Emma Zapata-Martelo, doctora en Sociología por la Universidad de Austin, Texas. Profesora e investigadora en estudios del Desarrollo Rural en el Colegio de Postgraduados, en Montecillo, Estado de México. Temas de especialización: sociología rural, género y mujer rural. Correo electrónico: emzapata@colpos.colpos.mx.
}

Julio Contreras-Utrera, doctor en Historia Contemporánea por la Universidad del País Vasco. Profesor en el Sistema de Enseñanza Abierta, región OrizabaCórdoba, de la Universidad Veracruzana. Temas de especialización: historia social, ambiental y económica. Correo electrónico: utrera63@hotmail.com. Benito Salvatierra-Izaba, doctor en Estudios del Desarrollo Rural por el Colegio de Postgraduados. Investigador titular B de El Colegio de la Frontera Sur. Temas de especialización: fecundidad, sobrevivencia infantil y salud reproductiva. Correo electrónico: bsalvati@ecosur.mx.

Enviado a dictamen: 02 de noviembre de 2012. Aprobación: 12 de diciembre de 2012. Revisiones: 1. 
Verónica Gutiérrez-Villalpando, Dominga Austreberta Nazar-Beutelspacher Emma Zapata-Martelo, Julio Contreras-Utrera, Benito Salvatierra-Izaba
MUJERES Y ORGANIZACIÓN SOCIAL EN LA GESTIÓN DEL AGUA PARA CONSUMO HUMANO Y USO DOMÉSTICO EN BERRIOZÁBAL, CHIAPAS

\section{Introducción}

$\mathrm{D}$ e acuerdo con Barkin (2006: 2), el sistema de gestión hídrica en México está en crisis aunque las autoridades aseguran que más de 90\% de la población tiene acceso al agua potable y que una parte un poco menor tiene conexiones al alcantarillado. Sin embargo, la realidad es que el país está sufriendo grandes carencias de agua por su inadecuada disponibilidad en calidad y cantidad. Este mismo autor menciona que son las mujeres, en particular, las que terminan soportando cargas desmedidas de trabajo por su responsabilidad como cuidadoras en la sociedad, destacando entre sus tareas el abasto de agua para sus hogares. Asimismo, se observan otros problemas, como la carga diferencial que recae sobre los grupos sociales más pobres por los elevados costos de comprar agua de pipas, o los grandes segmentos de la sociedad que se ven obligados a dedicar largas horas para acarrear, purificar, distribuir y desechar el agua. Barkin (2006: 4-5) ofrece una visión modificada de la nueva cultura del agua identificando los cuatro usos primordiales de ésta, cuya asignación debe reflejar prioridades diferenciales en el plano político: 1) agua como derecho humano; 2) agua para las necesidades ambientales; 3 ) agua para usos sociales y comunitarios; y 4) agua para el desarrollo económico. Este planteamiento tiene profundas implicaciones sociales y políticas que están plasmadas en la legislación mexicana. Es reconocido que el Estado mexicano tiene la obligación de asegurar que se cubran las necesidades básicas de la población, incluyendo el acceso al agua en cantidad y calidad adecuadas. Sin embargo, en México no existe capacidad institucional para ofrecer esta garantía y grandes segmentos de la población están marginados (Barkin, 2006).

Salvo en discursos retóricos, no hay un reconocimiento efectivo del papel activo que desempeña la población rural en la conservación y rehabilitación de las cuencas hidrológicas, ni de la necesidad de compensarla con incentivos por su aporte a la protección ambiental. Tampoco hay una apreciación real del daño a sus ambientes. Este autor menciona que los usos sociales y comunitarios del agua no son considerados en la legislación nacional (Barkin, 2006).

A pesar de ello, la gestión comunitaria ha sido identificada como un mecanismo para garantizar la gobernabilidad efectiva del agua a nivel local, especialmente en lo que atañe a los recursos de propiedad común. El concepto de gobernabilidad aplicado al agua se refiere a la capacidad social de movilizar energías en forma coherente para el desarrollo sustentable de los recursos hídricos. En dicha definición se incluye la capacidad de diseño de políticas públicas que sean socialmente aceptadas, orientadas al desarrollo sustentable del recurso hídrico, y de hacer efectiva su implementación por los diferentes actores involucrados. A menudo se asume que las instituciones locales son inclusivas y se ocupan de distribuir los recursos con justicia. Sin embargo, la realidad es que las comunidades están compuestas por categorías sociales distintas, como las de hombres y mujeres, en posiciones de poder diferentes, que aspiran a mejorar su propia situación. La gobernabilidad efectiva del agua tiene que incorporar un análisis diferenciado de la comunidad y la gestión comunal (GWA, 2003).

Para el caso del agua, la gestión en determinado territorio significa la toma de decisiones y la aplicación de prácticas locales para el establecimiento de normas y reglas, así como de obligaciones, sanciones y criterios de distribución y manejo del recurso hídrico (Boelens y Doornbos, 2001). Por su parte, Vera Delgado (2004) y Kabeer (1994) añaden al tema de la gestión las relaciones de poder, al señalar que la gestión es una construcción social que implica estrategias discursivas, confrontación y negociación. El poder se basa en la capacidad de mujeres y hombres para movilizar recursos y construir reglas. Diversos autores (Boelens, 2003; Barkin, 2004; Brooks, 2004; Gentes, 2004; Peña, 2004, en Galindo y Palerm, 2007) coinciden en la necesidad de identificar, revalorar y rescatar los conocimientos y técnicas locales que permiten a comunidades campesinas e indígenas su abasto de agua. También señalan que, además de reconocer sus formas organizativas, es necesario garantizar una participación efectiva que involucre tanto a hombres como a mujeres en la toma de decisiones para la gestión 
del agua, lo cual introduciría principios democráticos y equitativos en el acceso al agua y, además, se trataría de una medida para buscar el aprovechamiento sustentable del recurso en el nivel local (Boelens, 2003; Barkin 2004; Brooks, 2004; Gentes, 2004; Peña, 2004 en Galindo y Palerm, 2007).

De acuerdo con Galindo y Palerm (2007), en México y otros países se ha probado que en las zonas rurales el acceso y la gestión del agua, ya sea ésta para riego, consumo humano o consumo animal, tienen una estrecha relación con la presencia de técnicas locales, así como de instituciones y arreglos sociales de tipo comunitario/multicomunitario o entre usuarios del agua (Gelles, 1984; Agarwal y Narain, 1991; Boelens y Ávila, 1998; Elamon, 2005; Guzmán y Palerm, 2005; Galindo y Palerm, 2007). Estos autores mencionan que, a pesar de las voces que pugnan por revalorar los saberes y técnicas tradicionales para el abasto del agua y por el reconocimiento legal de la capacidad autogestiva que poseen las comunidades campesinas e indígenas para manejar sistemas hídricos, en México, con la Ley de Aguas Nacionales de 1992 y la creación de la figura de organismos operadores para la gestión del agua de consumo humano en las zonas rurales, el manejo del agua al parecer tiende a concentrarse, lo que puede o está teniendo efectos en las instituciones creadas y administradas por las mismas comunidades, de las cuales se desconoce tanto su funcionamiento técnico y social, como su permanencia a lo largo del tiempo y su eficiencia para lograr su objetivo: el abasto de agua (Galindo y Palerm, 2007).

\section{Gestión del agua y equidad de género}

México se caracteriza por un sistema marcado por las desigualdades en la disponibilidad, el acceso, el control y el uso del agua, así como por un uso ineficiente de este recurso. Recientemente ha incorporado en sus políticas elementos que provienen de los postulados formales de organismos internacionales tendentes a lograr la equidad de género en el acceso y control del agua, a hacer más eficiente su uso y a mejorar la calidad de vida de la población (Nazar et al., 2010).
No obstante los discursos de inclusión femenina en la gestión del agua, Ivens (2008) argumenta que, si bien la participación de las mujeres puede contribuir a mejorar la eficiencia en el manejo del agua para consumo humano, y aunque el suministro de agua por parte de ellas mejora su salud y la de sus hijos, no existe claridad acerca de cómo su participación, en particular en la toma de decisiones en el proceso de gestión, puede directamente contribuir a su bienestar personal, a su empoderamiento $y$, en última instancia, a la equidad de género. Más aún, a la poca claridad de los efectos de su participación, el mismo autor considera que, sin un cambio en los roles tradicionales de género, como el de las tareas domésticas, la participación de las mujeres en la gestión y manejo del agua comunitaria significará más horas de trabajo adicionales que se sumarán a las inequidades cotidianas que de por sí ya viven las mujeres.

Por suparte, Briadotti(2004), anteuncuestionamiento semejante, propone la necesidad de llevar a cabo programas simultáneos de equidad en el acceso a los recursos productivos como la tierra, el agua o las fuentes de empleo remuneradas dentro de un plan estratégico para generar un cambio en las relaciones de género en el interior y fuera del hogar. Nazar et al. (2010) mencionan que este último se trata de un elemento clave en la transformación institucional y en las relaciones sociales, aunque es difícil de instrumentar ya que conlleva, además de la redistribución del poder entre hombres y mujeres, la necesidad de repensar los esquemas de desarrollo que son responsables tanto del deterioro ambiental, como de la creciente desigualdad social.

\section{Problemática del abasto de agua en Chiapas. Contexto subcuencas Río Sabinal y Cañón del Sumidero}

En la perspectiva estadística, Chiapas en el año 2005 reportó que el $71.1 \%$ de las viviendas particulares habitadas tenía agua entubada (INEGI, 2005). En el censo de 2010, esta proporción aumentó a 73.5\% (INEGI, 2010). Estos datos muestran un mínimo incremento en este rubro (2.4\%), probablemente por debajo del crecimiento 
Verónica Gutiérrez-Villalpando, Dominga Austreberta Nazar-Beutelspacher Emma Zapata-Martelo, Julio Contreras-Utrera, Benito Salvatierra-Izaba
MUJERES Y ORGANIZACIÓN SOCIAL EN LA GESTIÓN DEL AGUA PARA consumo humano y uso doméstico en Berriozábal, Chiapas

\section{Área de estudio}

se quedan sin agua durante largos períodos, siendo las mujeres las más afectadas al verse obligadas a traerla desde otros manantiales sin que los hombres apoyen para cubrir esta necesidad. Pero la falta de agua también está asociada a la deforestación, la contaminación y la sobreexplotación de los mantos acuíferos. Ante esta situación se han instrumentado programas de ordenamiento territorial (POET) y planes de emergencia, como en el caso de la subcuenca Río Sabinal, la cual se ubica dentro de la Región Hidrológica 30 en la cuenca «E» Grijalva-Usumacinta, incluida en la subcuenca Tuxtla Gutiérrez-Río Sabinal (POET, 2010), y abarca los municipios de Tuxtla Gutiérrez, Ocozocoautla, Berriozábal y San Fernando en el estado de Chiapas.

Entre los problemas más comunes que han generado un impacto ecológico en esta subcuenca destacan: 1) la pérdida de espacios agroecológicos; 2) la ampliación de la mancha urbana; y 3) la contaminación de los cuerpos de agua a pequeña y gran escala por los efectos de la ganadería extensiva. Aunado a esto, existen problemas relacionados con la organización social en torno al recurso agua como son: 1) poca representatividad social; 2) frágil organización interna comunitaria; 3) mala comunicación entre los usuarios del agua; y 4) poca participación de la sociedad en general en la gestión integral del agua, sobre todo de las mujeres (POET, 2010).

Existen pocos estudios que analicen desde bases empíricas la organización social en la gestión comunitaria del agua y su impacto en la equidad y la sustentabilidad considerando las actuales políticas nacionales e internacionales sobre la gestión integral del recurso hídrico.

El propósito de esta investigación, realizada en las subcuencas Río Sabinal y Cañón del Sumidero en el estado de Chiapas, es analizar la magnitud y las características de la organización social en la gestión comunitaria del agua y el papel que juegan las mujeres dentro de ella. Se exploran algunos aspectos que favorecen o impiden la organización social en las comunidades que cuentan con comités de agua, así como también en las que carecen de ellos, y el papel que juegan las mujeres en ambos casos.
El municipio de Berriozábal, Chiapas, se localiza en la Depresión Central y colinda al norte con los municipios de Tecpatán y Copainalá, al este con Tuxtla Gutiérrez y San Fernando, y al oeste con el municipio de Ocozocoautla de Espinoza. Cuenta con una extensión territorial de 300.6 kilómetros cuadrados. Su altitud promedio es de 900 metros sobre el nivel del mar y se encuentra entre las coordenadas geográficas de latitud norte $16^{\circ} 48$ y de longitud oeste $93^{\circ} 16$. Se ubica dentro de la Región Hidrológica 30 en la cuenca «E» GrijalvaUsumacinta, incluida en la subcuenca Tuxtla GutiérrezRío Sabinal (POET, 2010). A esta subcuenca pertenece el río Sabinal objeto del presente estudio, que nace en la loma El Chupadero, a cinco kilómetros al noroeste del municipio de Berriozábal, a una altitud del orden de los 1100 metros sobre el nivel del mar, con dirección al sureste, y finalmente descarga en el río Grijalva, en las cercanías del Cañón del Sumidero, aportando a éste un área de drenaje de 375 kilómetros cuadrados aproximadamente. Su importancia radica en que en su cauce se asienta la principal zona urbana del estado de Chiapas, la ciudad de Tuxtla Gutiérrez.

El municipio de Berriozábal tiene una población estimada de 43179 habitantes, con una población masculina de 21562 habitantes y una población femenina de 21617 . Existe una población analfabeta de 4745 habitantes, de la cual $37.9 \%$ corresponde a hombres y $62.1 \%$ a mujeres. La población mayor de 15 años con primaria incompleta se reporta en 6597 personas, de las cuales $48.2 \%$ son hombres y $51.8 \%$ son mujeres. La población mayor de 15 años con primaria completa se reporta en 5216 habitantes; con secundaria incompleta, 1406 personas; con secundaria completa, 4172; y con educación posbásica, 4958. En cuanto a la población económicamente activa, se reportan 15665 personas. De las 9242 viviendas reportadas, $56.7 \%$ dispone de agua entubada y $42.8 \%$ no dispone de ella. En el caso del servicio de electricidad, solamente 10\% de la población no cuenta con el servicio. De la población total, $48.4 \%$ se reporta sin derechohabiencia y $49.6 \%$ sí la tiene. 
Verónica Gutiérrez-Villalpando, Dominga Austreberta Nazar-Beutelspacher Emma Zapata-Martelo, Julio Contreras-Utrera, Benito Salvatierra-Izaba
MUJERES Y ORGANIZACIÓN SOCIAL EN LA GESTIÓN DEL AGUA PARA CONSUMO HUMANO Y uSO DOMÉstiCo EN BERRIOZÁBAL, CHIAPAS

\section{Universo y diseño de estudio}

El universo del presente estudio comprendió a las 55 comunidades rurales y urbanas marginales ubicadas en las subcuencas Río Sabinal y Cañon del Sumidero en el municipio de Berriozábal, Chiapas, México (mapa 1). De las 55 localidades, 49 corresponden a comunidades rurales y seis a asentamientos urbanos marginales. En el levantamiento de datos, sólo se incluyeron 46 de estas 55 comunidades - en unos casos no se tuvo acceso por malas condiciones de los caminos, dado que el trabajo se desarrolló en época de lluvias, y en otros casos se trata de comunidades deshabitadas-.

El trabajo de campo se realizó en los meses de mayo, junio y julio de 2011 con los objetivos de: a) identificar las fuentes de abastecimiento de agua para consumo doméstico; b) identificar la infraestructura de abastecimiento; c) conocer la organización social en torno a los comités de agua comunitarios; y d) conocer la existencia de otros comités comunitarios como referente de su organización social.

Los instrumentos a partir de los cuales se obtuvo la información fueron: 1) observación participante; 2) aplicación de un cuestionario estructurado con preguntas sobre los comités comunitarios existentes en cada localidad, en particular los relacionados con la gestión del agua, el cual incluyó ítems sobre quiénes los conforman y qué cargos desempeñan; y 3) entrevistas en profundidad a actores clave.

El espacio muestral estuvo compuesto por el total de comités comunitarios ( $\mathrm{N}$ ) de las 46 comunidades en las que se trabajó, las cuales están distribuidas en las dos subcuencas de estudio. De esta manera, la unidad de análisis estuvo constituida por cada uno de los comités de agua de cada comunidad (n), o cuales se aplicaron los instrumentos de levantamiento de datos. Una vez determinada la presencia/ausencia y tipo de comités comunitarios - de acuerdo con el tipo de gestión social que realizan, como agua, luz, salud o escuelas-, las comunidades se clasificaron según presencia y ausencia de comités de agua en dos condiciones: a) aquellas con comité de agua ( $\mathrm{y}=1)$ y b) aquellas sin comité de agua ( $\mathrm{y}=2)$. De esto resultó que sólo en 24 comunidades existen comités de gestión del agua (n), mientras que se registró su ausencia en las 22 restantes.

Para realizar el análisis de la información generada en el estudio de campo, se construyó una base de datos con variables de caracterización socioeconómica de (n) a partir del Censo General de Población y Vivienda del INEGI de 2010. La base de datos incluyó variables agrupadas en dos categorías: educación y vivienda/ servicios. A partir de ellas, se realizó una comparación de medias y una prueba de análisis de varianza de una sola vía entre las comunidades de cada una de las dos condiciones $(y=1, y=2)$, con el objetivo de conocer si se sugería alguna relación entre dichas variables y la existencia de alguno de los dos tipos de condiciones. Los resultados reportaron el valor de probabilidad de error tipo I o alfa $(\alpha)$ y se utilizó la prueba de homogeneidad de varianzas de Levene según Daniel (2010). En las variables socioeconómicas para las que la prueba de Levene mostró heterocedastocidad de varianzas, se utilizó la prueba de Games-Howell, siguiendo de nuevo a Daniel (2010) para la comparación múltiple. En el caso de las variables en las que la prueba de Levene mostró varianzas iguales, se utilizó la diferencia mínima significativa (DMS), siempre según Daniel, para la comparación múltiple. Con base en la información de tipo cualitativo, los testimonios de las entrevistas a profundidad realizadas fueron clasificados en dos temas: 1) organización social y problemática en la autogestión del agua en las comunidades con comité de agua, y 2) organización social y problemática en la autogestión del agua en las comunidades sin comité de agua.

\section{Resultados}

Se encontraron 16 tipos de comités comunitarios. Los comités con mayor frecuencia fueron los siguientes: escuelas primarias (18.8\%), consejos de vigilancia (18.4\%), comités del programa Oportunidades de la Secretaría de Desarrollo Social (14.31\%), agua (12.7\%), salud (12.4\%) y desayunos escolares (10.1\%). Los comités con menor frecuencia fueron: Comité de Planeación para el Desarrollo Municipal (COPLADEM) (3.5\%), luz (2.7\%), 
Programa Alimentario de la Secretaría de Desarrollo Social (1.9\%), preescolar (1.6\%), participación ciudadana (0.7\%) y Chiapas Solidario de la Secretaría de Desarrollo y Participación Social (0.7\%). Además, se reportaron con $0.4 \%$ cada uno de los siguientes comités: Sociedad de Solidaridad Social Ganadera, cocinas comunitarias y escuelas secundarias. De los 16 tipos de comités encontrados en las localidades de las subcuencas Río Sabinal y Cañón del Sumidero, solamente cinco corresponden a comités de atención social — Programa Alimentario, salud, programa Oportunidades de la Secretaría de Desarrollo Social, desayunos escolares y escuelas secundarias-, los cuales están conformados en su mayoría por mujeres y, de éstos, solamente en dos sus integrantes son exclusivamente del sexo femenino. Estos dos comités, que corresponden a programas sociales a cargo de la Secretaría de Desarrollo Social, son Oportunidades y el Programa Alimentario.

En los comités que abordan actividades productivas observamos una mayor participación masculina; por ejemplo, los comités de la Sociedad de Solidaridad Social Ganadera y Agropecuaria están compuestos exclusivamente por hombres. Asimismo, en el caso del COPLADEM, el 99\% de los participantes son hombres y solamente el $1 \%$ de mujeres.

\section{Ubicación geográfica de los tipos de comités} basados en la organización comunitaria en torno a la gestión del agua

En materia de organización social en torno a la gestión del agua, a lo largo de la zona de estudio se encontraron 24 comités de agua, distribuidos en el mismo número de comunidades, donde se presenta la condición y=1 en $52.2 \%$ de las comunidades. En 22 comunidades no existe comité de agua, de tal manera que equivalen a un $47.8 \%$ del total las comunidades que representan la condición $\mathrm{y}=2$.

De las 24 comunidades que tienen comité de agua, 15 de ellas están ubicadas en la subcuenca Río Sabinal y nueve en la subcuenca Cañón del Sumidero. En la tabla 1 puede observarse que de las 22 comunidades que no cuentan con comité de agua, 17 están ubicadas en la subcuenca Río Sabinal y cinco en la subcuenca Cañón del Sumidero, por lo que la organización comunitaria en la gestión del agua no presenta una distribución particular en ninguna de las subcuencas. Sin embargo, una cuenca es un espacio hidrográfico y tal vez haya alguna relación con la hidrografía o con la hidráulica que no fue analizada en este estudio.

\section{Factores explicativos de la existencia o no de comités de agua en las comunidades}

En términos generales, se aprecia que son las condiciones comunitarias de mejores servicios, incluyendo la derechohabiencia de la población, las que explican la existencia de comités de agua en las comunidades. Destaca, desde luego, el mayor porcentaje de viviendas con agua entubada (64.2\%, p=0.000), la mayor proporción de hogares con electricidad $(92.2 \%, \mathrm{p}=0.021)$ y con pisos de recubrimiento $(74.3 \%, \mathrm{p}=0.057)$, y marginalmente significativa fue la proporción de personas que son derechohabientes (54.7\%, $\mathrm{p}=0.061)$ (ver tabla 2 ).

Las comunidades con mayor desventaja en condiciones de vivienda, servicios y equipamiento se ubican entre las que no cuentan con comité de agua, lo que sugiere menor organización pese a que la gestión organizada del agua y la participación de las mujeres en los comités serían elementos clave para promover el desarrollo sustentable con equidad. Es notorio que en aquellas comunidades que no cuentan con agua entubada no existe comité de agua, ya que éstos son establecidos por el gobierno como una obligación, cuando se realiza la obra de agua entubada. No son comités que nacen de las bases, aun cuando éstos podrían ser fundamentales para la gestión de la dotación.

\section{Problemática y organización social en las localidades que cuentan con comité de agua comunitario}

La existencia de comités de agua comunitarios se asocia directamente con la presencia de agua entubada, aunque en las comunidades sin comité de 
agua la responsabilidad de su abastecimiento para el hogar recae principalmente en las mujeres. En sus reglamentos tienen establecida una cuota fija mensual que varía entre cinco y 30 pesos. El monto de la cuota se determina en asamblea comunitaria en el caso del área rural y este aporte es utilizado para pagar el recibo de luz de la bomba de agua, a la persona encargada de bombearla, comúnmente llamada "bombero del agua", y el mantenimiento o arreglo de tuberías. También tienen definido su sistema de sanciones en caso de que algún usuario no quiera pagar. Algunas de estas sanciones son: 1) baja del padrón de beneficiarios de agua; 2) sellado de la toma de agua; 3) pago de multas; o 4) dar un plazo para que pague y, en caso contrario, se le corta el servicio.

Algunos de los problemas que se han presentado en la autogestión del agua en las comunidades donde existe comité de agua son los siguientes: 1) falta de pago de recibos de luz — que utilizan en el bombeo de agua - al ayuntamiento; 2) multas por parte de la Comisión Federal de Electricidad; 3) carencia de agua, principalmente en época de sequía; 4) pozos con agua insuficiente para abastecer el crecimiento poblacional de algunas comunidades; 5) necesidad de mejorar la infraestructura; por ejemplo, la necesidad de un tanque más grande para almacenar el agua y una mejor red de distribución; 6) la comunidad no es dueña de la vertiente de agua; y 7) en algunas comunidades, aumento de las horas de bombeo en época de sequía, lo que se ve reflejado en un aumento del recibo de luz.

Algunas comunidades de las subcuencas Río Sabinal y Cañon del Sumidero, a pesar de contar con agua entubada y comité de agua, realizan estrategias para abastecerse del líquido y ahorrar agua en época de sequía debido a la reducción de la cantidad de agua entubada para consumo humano y uso doméstico en esta época, lo que concuerda con lo encontrado por Soares (2006) en la comunidad de Pozuelos, municipio de San Juan Chamula, donde, según menciona, hay meses del año en los cuales el agua escasea, lo cual se asocia a la temporada de sequía y a la necesidad del abasto directo en las fuentes.
En el caso de las comunidades ubicadas en las subcuencas Río Sabinal y Cañón del Sumidero, podemos afirmar que el abasto de agua es estacional y está restringido a los meses de estiaje dado que las comunidades cuentan con un sistema de abastecimiento de agua. Lo anterior concuerda con lo encontrado por Soares (2006), quien al respecto menciona que la llave en el hogar es la fuente más importante de dotación de agua para las familias; sin embargo, en el periodo de estiaje, que puede durar de dos a seis meses, el acarreo de agua es la principal forma de acceso al agua por parte de las unidades domésticas (Soares, 2006). Sin embargo, en Berriozábal encontramos que las mujeres son las principales responsables de realizar este acarreo de agua.

Los habitantes de las subcuencas Río Sabinal y Cañón del Sumidero en el municipio de Berriozábal ponen en práctica algunas estrategias para abastecerse del líquido en época de sequía; por ejemplo: 1) lavan ropa o trastes y se bañan en los ríos o vertientes cercanos, lo que puede ocasionar la contaminación del agua por uso de detergentes, o enfermedades gastrointestinales por el consumo de agua contaminada, y 2) acarrean el agua de los ríos, pozos o vertientes cercanas a sus casas. Esta actividad generalmente la realizan mujeres y niños.

En algunas comunidades que cuentan con comité de agua, la vertiente o manantial de agua se encuentra en propiedad privada o en otra comunidad. A continuación se cita un testimonio al respecto:

[...] Tenemos un poco de problemas con el dueño del terreno donde está el manantial porque ya se venció el plazo que teníamos para sacar agua (Víctor, presidente del patronato del agua, El Divizadero).

\section{Problemática y organización social en las comunidades sin comité de agua}

Los problemas principales que encontramos en las comunidades que no cuentan con agua entubada y tampoco con comité de agua son: 1) acarreo permanente 
de agua en algunas comunidades, y en otras solamente en época de sequía, responsabilidad que recae principalmente en las mujeres y los niños; 2) ir al río a lavar ropa y trastes, bañarse y acarrear agua para tomar; 3) falta de organización comunitaria para abastecerse de agua, que por lo general se realiza de manera individual o a nivel de familia; y 4) en el caso de las comunidades que no cuentan con una vertiente cercana, pago de altos costos por pipas de agua.

En su mayoría, los habitantes de las comunidades sin comité de agua la acarrean de algunas vertientes o arroyos cercanos, aunque algunos de éstos se encuentran a más de un kilómetro de distancia. También encontramos algunas comunidades que tienen mangueras conectadas a estas vertientes, $y$ otras comunidades urbano-marginales y rurales cercanas a la cabecera municipal de Berriozábal que tienen que comprar pipas de agua. En algunos casos, las pipas se las proporciona el Sistema de Agua Potable y Alcantarillado Municipal de Berriozábal, pero en su mayoría compran a empresas particulares donde la pipa de 3000 litros tiene un costo de 150 pesos.

A diferencia de la gestión comunitaria que se realiza en las comunidades con comité de agua, en las comunidades que no cuentan con este comité la gestión se realiza generalmente de manera individual o familiar y esta responsabilidad recae principalmente en las mujeres. En este sentido, el tema central parece ser la división tradicional por géneros del trabajo, porque, como se ha enunciado, la participación de las mujeres en los comités de agua comunitarios, que implica trabajo extradoméstico y recorridos extensos fuera de su vivienda —en el ámbito público-, no es aceptada por las comunidades:

Solamente los hombres participamos en los comités de la comunidad por motivo de que en ocasiones hay que salir fuera de la comunidad y no se ve bien que otra mujer que no sea su pareja vaya con él. Si alguna mujer participara en el comité de la comunidad sería más conveniente que en este comité participara la pareja para que no hubiera malos entendidos o comentarios (Crispín, agente rural, ejido Cuchumbac).
Por el contrario, la gestión del agua en el interior del hogar, aunque beneficia a todos los integrantes de las familias, también recae en las mujeres, a quienes se considera responsables de obtenerla debido a la división sexual del trabajo. El acarreo del agua no es considerado propiamente un trabajo y forma parte de la no valoración del trabajo doméstico en su conjunto. Desde los enfoques de género y equidad, la responsabilidad de la gestión del agua debería ser compartida por hombres y mujeres. Los siguientes fragmentos dan cuenta de estas consideraciones:

En esta comunidad el agua lo tienen que acarrear las mujeres con botes sobre la cabeza, ya que lo hacen en el transcurso del día y en ese rato los hombres estamos trabajando y es por ello que no acarrean los hombres. Hemos solicitado agua entubada ante el municipio, pero no hemos tenido respuesta (Reynaldo, suplente agente rural, ranchería Berling).

\section{Reflexiones sobre la organización comunitaria de la gestión del agua y papel que juegan las mujeres}

Actualmente, diversos autores coinciden en señalar que la participación ciudadana en los procesos de toma de decisiones, ligada a la organización comunitaria, contribuye a una mejor gestión del agua, lo cual se considera una estrategia para lograr el desarrollo sustentable en el ámbito local (Ostrom, 1990; Córdova et al., 2006; Delgado et al., 2007; Galvin y Haller, 2008).

En este sentido, fue importante analizar la gestión del agua en las subcuencas Río Sabinal y Cañon del Sumidero. Como grupos organizados a través de un comité de agua comunitario, los ejidatarios y usuarios del agua tienen el control y administran el agua, su organización es autogestiva, actúan con recursos propios y no interviene el gobierno en la toma de decisiones, lo que concuerda con lo encontrado por De León (2006) en las comunidades El Jalocote y La Lima en Autlán, Jalisco. Asimismo, presentan semejanzas con los comités de agua potable independientes de Toluca, estado de México, debido a que hay una presencia alta de cooperación en el ámbito comunitario al llevarse a 
Verónica Gutiérrez-Villalpando, Dominga Austreberta Nazar-Beutelspacher Emma Zapata-Martelo, Julio Contreras-Utrera, Benito Salvatierra-Izaba
MUJERES Y ORGANIZACIÓN SOCIAL EN LA GESTIÓN DEL AGUA PARA CONSUMO HUMANO Y USO DOMÉSTICO EN BERRIOZÁBAL, CHIAPAS cabo diversas acciones con usuarios de agua potable. Sin embargo, existe poca e incluso nula comunicación de los comités de agua de las comunidades con las instituciones gubernamentales y entre ellos mismos. Solamente establecen relaciones con el ayuntamiento cuando los comités de agua no pueden cubrir la totalidad del pago de la energía eléctrica consumida, o cuando es necesario comprar una bomba de agua, la ampliación de la red hidráulica o la construcción de cárcamos. Pero esta relación es puntual y sólo se hace referencia a la solicitud de apoyos económicos y permisos, lo que también concuerda con lo que ocurre en la mayoría de los comités de agua de Toluca.

En aproximadamente la mitad de las comunidades ubicadas en las subcuencas Río Sabinal y Cañón del Sumidero existe una carencia de agua entubada, la cual se relaciona directamente con la ausencia de comités comunitarios de agua. En estas comunidades las mujeres juegan un papel muy importante en la organización social en la gestión del agua. Como pudimos observar, son ellas quienes proporcionan prácticamente toda el agua que se necesita para consumo humano y uso doméstico.

En general, tanto en los ámbitos rurales como urbano-marginales de este estudio, la responsabilidad femenina en estas tareas es mayor debido a que la cantidad de agua dulce disponible en las fuentes actualmente ya no es suficiente, principalmente en época de sequía, y también debido al aumento de población, lo que ha ocasionado que tengan que recurrir a los ríos o arroyos más cercanos para abastecerse de este líquido y realizar actividades como lavar ropa, trastes, bañarse y acarrear agua para consumo humano.

Sin embargo, la marcada segregación sexual en el uso del agua, así como en las actividades productivas para las cuales es un insumo fundamental, origina diferencias y desigualdades entre mujeres en cuanto al acceso, uso, manejo y control de los recursos hídricos, y tiene como corolario la baja incidencia de las mujeres en las instancias de toma de decisiones relacionadas con el agua, como por ejemplo en los comités de agua comunitarios en Berriozábal, que están conformados por habitantes locales, en su mayoría hombres, encargados de administrar, manejar, detectar y reparar fallas en la red de abastecimiento de agua, generalmente con mandato por un año (Gutiérrez et al., 2012). Lo anterior concuerda con lo encontrado por Soares (2003) en la comunidad tsotsil de Pozuelos del municipio de San Juan Chamula, quien afirma que, en principio, todos los habitantes de la comunidad pueden acceder al agua, aunque el control y las decisiones relacionadas con la gestión del recurso hídrico son asumidos por los hombres que conforman el patronato del agua. De acuerdo con Soares (2003), la existencia de una organización que regula el acceso al agua para uso doméstico, con representantes exclusivamente del sexo masculino, en una comunidad donde tanto el abasto como el manejo del agua en la unidad doméstica son responsabilidad casi exclusiva de las mujeres, refleja que los valores sociales y los estereotipos culturales son los que guían las prácticas comunitarias de organización y gestión de los recursos. La situación de exclusión de las mujeres en los espacios de toma de decisiones en relación con el agua para uso doméstico no es privativa de Pozuelos, sino que la comparten diferentes regiones de Chiapas, como pudimos constatar en algunas comunidades de Berriozábal.

Al respecto, González Montes (1997), Moser (1995) y Siles y Soares (2003), entre otras autoras, sugieren que las mujeres, como categoría social, tienen acceso desigual a los recursos y al poder, y que estas desigualdades constituyen un gran nudo para lograr la sustentabilidad a nivel micro, toda vez que el desarrollo sustentable no se refiere exclusiva o fundamentalmente a un cierto uso y manejo de los recursos naturales, sino también a una dimensión humana que involucra la calidad de vida de los sujetos sociales y el empoderamiento de las mujeres.

Según lo planteado por Kauffer y García (2003), de los 1000 comités o patronatos de agua distribuidos en todo Chiapas, sólo diez cuentan con presencia femenina, es decir, apenas el 1\%, lo que confirma la baja participación de las mujeres en los espacios públicos.

En estos términos, son las mujeres quienes conocen más que nadie las demandas reales de las unidades domésticas en lo tocante al agua, por la sencilla razón 
de que son las responsables de manejar el recurso dentro del hogar, ya sea en el lavado de ropa, la preparación de alimentos o el aseo de la vivienda, entre otras actividades. De acuerdo con Soares (2003), son los hombres quienes determinan las prioridades para utilizar el agua en las unidades domésticas y también quienes establecen las sanciones para los incumplimientos. Son ellos quienes deciden, incluso, en aspectos relacionados con actividades eminentemente femeninas.

Al respecto, Paolisso y Ramírez (2003) y Soares (2006) afirman que trabajos de investigación recientes apuntan que en las unidades domésticas pobres las mujeres están más motivadas que los hombres para conservar y administrar los recursos naturales locales, esto porque son ellas las responsables de la satisfacción de las necesidades de alimentación y salud de sus hogares, y dicha responsabilidad se cumple de manera más adecuada si cuentan con suficientes recursos, como por ejemplo agua y leña.

\section{Conclusión}

Desde la perspectiva de los derechos humanos a los que apelan los movimientos de defensa del agua, entre ellos la Agenda Azul, el agua es un bien común y su acceso es un derecho inalienable y fundamental. Hoy en día la Constitución Política de los Estados Unidos Mexicanos reconoce de manera expresa el derecho humano de toda persona al acceso, disposición y saneamiento de agua para consumo personal y doméstico en forma suficiente, saludable y asequible (Reforma al artículo $4^{\underline{0}}$ Constitucional, DOF, 8 de febrero de 2012). En ese sentido, el Estado debe cumplir su rol de garante y está obligado a crear las condiciones para que todas las personas, tanto hombres como mujeres, puedan participar en las políticas relacionadas con el acceso a los recursos hídricos y su gestión. Esto implica que la privatización del agua no es la vía y sí lo es la reestructuración de las instancias que tienen que ver con el recurso. El proceso de sensibilización y la creación de espacios en las instituciones gubernamentales y comunitarias son urgentes para la formulación de políticas y programas con enfoque de equidad de género, así como la modificación de la visión sobre las mujeres, quienes tradicionalmente han sido beneficiarias o consumidoras del agua, y no administradoras ni tomadoras de decisiones.

La relación entre pobreza y agua es contundente sobre todo en los ámbitos rurales, y esto resulta en una severa contradicción al verificarse que las mujeres no forman parte del proceso de toma de decisiones en relación con el agua, tratándose ésta de un bien social en cuyo cuidado y conservación cumplen un papel primordial. En este caso, si las políticas públicas siguen desvinculadas de la sociedad se profundizará la feminización de la pobreza y continuarán los problemas de escasez de recursos hídricos.

En las comunidades de estudio, independientemente de que dispongan o no de comités, se observa que la separación entre lo público y lo privado, aunque ambigua en muchas instancias, refuerza la concepción de que las tareas relacionadas con el agua destinada para uso doméstico, como el acarreo de agua al interior del hogar, son mencionadas como actividades propias de las mujeres en oposición al carácter masculino de la participación en los comités comunitarios de agua.

En los testimonios de las entrevistas realizadas a 37 hombres se manejan discursos que "colectivizan" los problemas y las estrategias de resolución entre hombres y mujeres, aunque en la práctica, y desde la visión de las entrevistadas - 18 mujeres-, son ellas quienes culturalmente asumen el rol de asegurar el abasto y el manejo del líquido vital en sus hogares sin que sus necesidades, saberes y experiencias de la vida cotidiana sean considerados como insumos fuera del ámbito privado. Las responsabilidades que se asignan a las mujeres para los servicios de saneamiento no son posibles bajo un contexto de inequidad; la sola demanda de acceso al agua potable y al saneamiento para aliviar las cargas de trabajo de las mujeres es insuficiente, pues deja intocada una división sexual del trabajo y una organización social que causa y reproduce las desigualdades entre hombres y mujeres.

No es deseable que las mujeres, junto con niños y niñas, sigan siendo las únicas o principales responsables del aprovisionamiento del agua en cantidad y calidad 
suficientes para las tareas domésticas, sino que las políticas para proporcionar acceso al agua deben acompañarse de medidas que fomenten un reparto equitativo entre hombres y mujeres, tanto del trabajo reproductivo como del productivo, en igualdad de oportunidades y condiciones. Asimismo, las políticas hídricas deben reformularse a la par de otras, como las de reforestación, agrícolas y sociales, para promover la participación equitativa.

Lo anterior conlleva una reflexión de tipo más teórico en la que se discuta de qué manera se puede dialogar con el Estado, cuyas políticas, en su visión más amplia, están profundamente masculinizadas. Así, se propone la realización de una revisión de la legislación ambiental en la materia desde una perspectiva de género, en particular de la Ley de Aguas Nacionales, para incidir en la política pública ambiental, lo cual podrá incluir, entre otras medidas, el establecimiento de cuotas de participación por sexo en los comités o patronatos de agua (Soares, 2006).

También es importante resaltar que la participación de las mujeres en la planeación, introducción y mantenimiento de los servicios de suministro y tratamiento de agua aumentaría sus posibilidades de empleo asalariado, aunque sabemos que esto implica la remoción de barreras culturales y su acceso a capacitación técnica en actividades como éstas, que muestran una pronunciada masculinización.

Finalmente, mientras algo de lo anterior sucede, serán bienvenidas las propuestas de participación ciudadana que favorezcan la coalición social para temas ambientales y que propugnen una relación más justa y equitativa para las mujeres. Para que los planteamientos de las organizaciones sociales y de las asociaciones civiles se conviertan en políticas públicas, sus acciones tendrían que dejar de ser puntuales y aisladas. Asimismo, el Estado tendría que dejar a un lado la resistencia para abrir espacios de participación social en la definición, orientación, implementación y evaluación de políticas públicas.

Por lo tanto concluimos que, si se tomara en cuenta la visión de las mujeres sobre el abasto y manejo cotidiano del agua, se fortalecería la organización comunitaria en torno a la gestión del agua para el uso doméstico y el diseño de las políticas públicas sería adecuado, equitativo en términos sociales, y sustentable en términos ambientales y económicos.

\section{Agradecimientos}

Al Consejo Nacional de Ciencia y Tecnología por la beca de posgrado otorgada para realizar el presente trabajo.

\section{Bibliografía}

Barkin, David (2004), "La gestión popular del agua: respuestas locales frente a la globalización centralizadora", en Futuros, núm. 7, vol. III, 2004.

Barkin, David (coord.) (2006), La nueva gestión del agua urbana en México: retos, debates y bienestar, México: Universidad de Guadalajara/ANEAS.

Boelens, Rutgerd y Patricia Ávila (eds.) (1998), Buscando la equidad: concepciones sobre justicia y equidad en el riego campesino, Países Bajos: Von Goreum \& Comp.

Boelens, Rutgerd (2003), "La lucha indígena por el agua y las políticas culturales de la participación”, Ponencia presentada en el III Foro Mundial del Agua, Sesión: Agua y Diversidad Cultural, marzo de 2003, Kioto, Japón.

Braidotti, Rosi (2004), "Mujeres, medio ambiente y desarrollo sustentable: surgimiento del tema y diversas aproximaciones", en Verónica Vázquez G. y Margarita Velázquez G. (comps), Miradas al futuro. Hacia la construcción de sociedades sustentables con equidad de género, México: PUEG/CRIM/CP/IDCR, pp. 23-59.

Brooks, David (2004), Agua: manejo a nivel local, Colombia: Alfaomega.

Daniel, Wayne W. (2010), Bioestadística: base para el análisis de las ciencias de la salud, México: Limusa Wyley.

Galindo Escamilla, Emmanuel y Jacinta Palerm Viqueira (2007), "Pequeños sistemas de agua potable: entre la autogestión y el manejo municipal en el estado de Hidalgo", en Agricultura, Sociedad y Desarrollo, vol. 4, núm. 2, pp. 27-145.

Galindo Escamilla, Emmanuel y Jacinta Palerm Viqueira (2009), "Las otras instituciones y el manejo eficiente del agua potable a pequeña escala: el Valle Tlazintla- 
Pozuelos, un caso de autogestión", Ponencia XXXIV Simposium de Historia y Antropología, edición internacional Tierra y agua: protagonistas de la historia Hermosillo, Sonora, 23 al 27 de febrero.

Gelles, Paúl (1984), Agua, faenas y organización comunal en los Andes: el caso de San Pedro de Casta, (tesis de maestría), Pontificia Universidad del Perú, Perú.

INEGI (2005), Conteo de población y vivienda, México: Instituto de Estadística, Geografía e Informática.

INEGI (2010), Censo general de población y vivienda, México: Instituto de Estadística, Geografía e Informática.

Ivens, Saskia (2008). "Does increased water access empower women?", en Development, núm. 5l, pp. 63-67.

Kauffer E. y García G.A. (2003) "Mujeres en los comités de agua del estado de Chiapas: elementos para entender una participación con segregación genérica", en Tunón Pablos, Esperanza (coord.) Género y Medio Ambiente, Ecosur -Semarnat- Plaza y Valdes, México.

Nazar Beutelspacher, Austreberta Dominga, Emma Zapata Martelo y Verónica Ramírez Castel (2010), "Género y agua. Reflexiones sobre las estrategias internacionales para lograr la sustentabilidad con equidad", en Blanca Jiménez, María Luisa Torregrosa y Luis Aboites Aguilar (eds.), El agua en México: cauces y encauces, México: Academia Mexicana de Ciencias/

Comisión Nacional del Agua, pp. 383-410.

POET (Programa de Ordenamiento Territorial de la subcuenca Río Sabinal en los municipios de San Fernando, Berriozábal, Ocozocoautla de Espinoza y Tuxtla Gutiérrez) (2010), Periódico Oficial, núm. 223, Tuxtla Gutiérrez: Secretaría General de Gobierno, pp. 135-191. 〈http://www.semarnat.gob. $\mathrm{mx} /$ temas/ordenamientoecologico/Documents/ documentos\%20decretados/decretos_2010/decreto_ sabinal.pdf> [1 de diciembre de 2010].

Ruíz Meza, Laura Elena (2012), "Relaciones de género y mercados de derechos de agua y tierra en Chiapas", en Región y Sociedad, vol. XXIV. 〈http://www.redalyc. org/src/inicio/ArtPdfRed.jsp?iCve=10223040002> [19 de octubre de 2012].

Siles, Jackeline y Denise Soares (2003), La fuerza de la corriente: gestión de cuencas hidrográficas con equidad de género, San José de Costa Rica: Hivos/UICN.

Soares, Denise (2006), "Mujeres, agua, leña y desarrollo: estudio de caso sobre género y recursos naturales en los Altos de Chiapas", en Verónica Vázquez et al. (coords.), Gestión y cultura del agua, t. II, México: IMTA/ COLPOS, pp. 293-312. 
Tabla 1. Número total de comunidades con presencia/ausencia de comités de agua

\begin{tabular}{|c|c|c|c|}
\hline & Cañón del Sumidero & Río Sabinal & Total \\
\hline Total de comunidades & 14 & 32 & 46 \\
\hline Total de habitantes & 4944 & 3852 & 8796 \\
\hline Total de comités comunitarios & 83 & 61 & 144 \\
\hline Total de comunidades con comité de agua & 9 & 15 & 24 \\
\hline Total de comunidades sin comité de agua & 5 & 17 & 22 \\
\hline
\end{tabular}

Fuente: elaboración propia con base en trabajo de campo, 2011.

Tabla 2. Características de las comunidades de acuerdo con su condición de existencia/ausencia de comités de agua

\begin{tabular}{||l||c||c||c||}
\hline \multicolumn{1}{|c|}{ Variables } & $\begin{array}{c}\text { Valores promedio de } \\
\text { comunidades para la } \\
\text { condición } \mathbf{y = 1} \text { (con comité } \\
\text { de agua } \mathbf{y} \text { participación de } \\
\text { las mujeres) (n=24) }\end{array}$ & $\begin{array}{c}\text { Valores promedio de } \\
\text { comunidades para la } \\
\text { condición } \mathbf{y = 2} \text { (sin comité } \\
\text { de agua) (n=22). }\end{array}$ & $\begin{array}{c}\text { Valor } \mathbf{p} \\
\text { Derechohabiencia }\end{array}$ \\
\hline \hline Con electricidad & 54.71 & 40.12 & 0.061 \\
\hline \hline Con agua dentro de la vivienda & 92.19 & 67.58 & 0.021 \\
\hline \hline Con drenaje & 64.21 & 15.66 & 0.000 \\
\hline \hline Con piso firme o recubrimiento & 72.82 & 59.25 & 0.178 \\
\hline \hline
\end{tabular}

Fuente: elaboración propia con base en datos del Censo General de Población, INEGI (2010). 
Mapa 1. Mapa de las comunidades ubicadas en la subcuenca Río Sabinal y Cañón del Sumidero

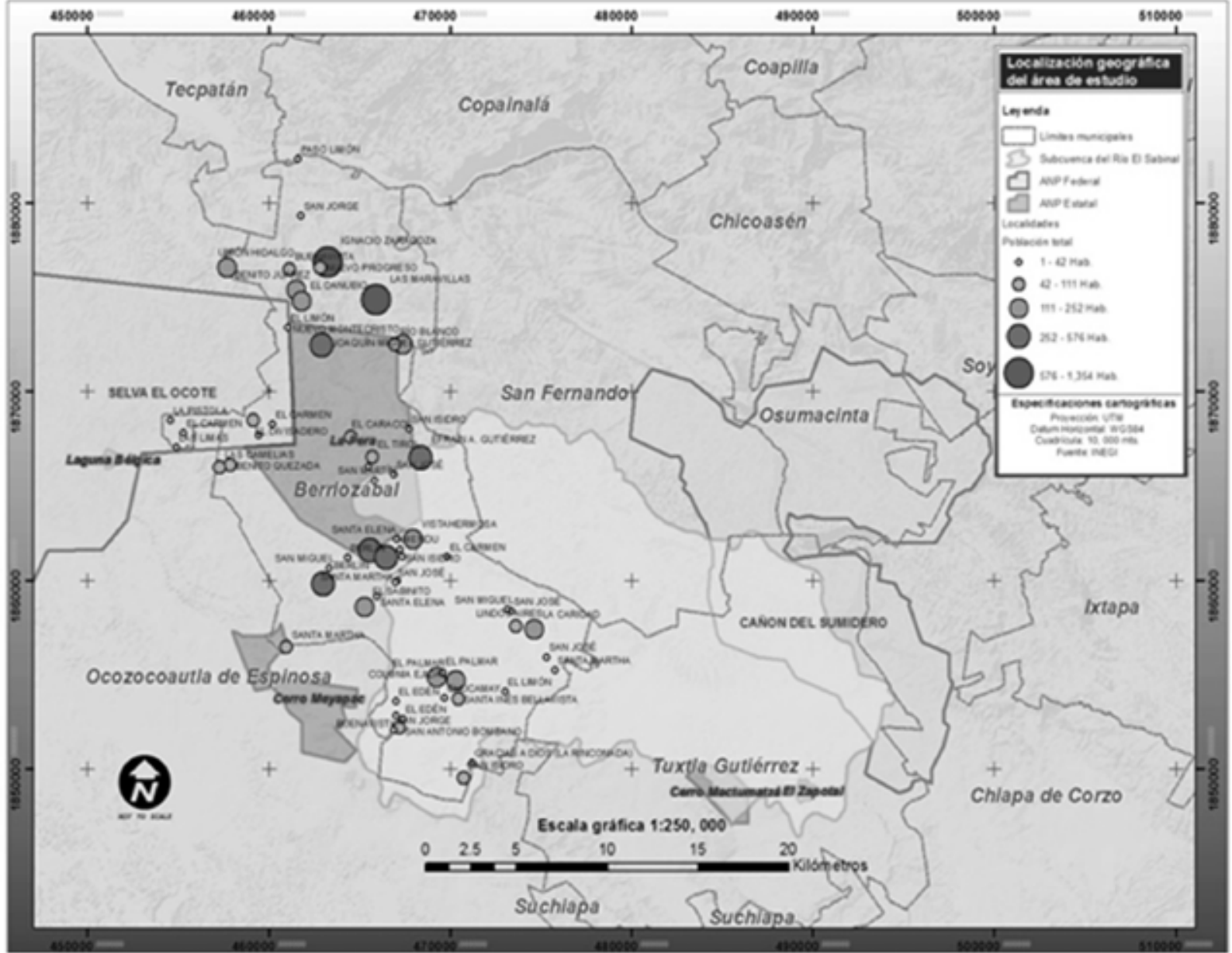

Fuente: elaboración propia con base en recorrido de campo, 2011. 